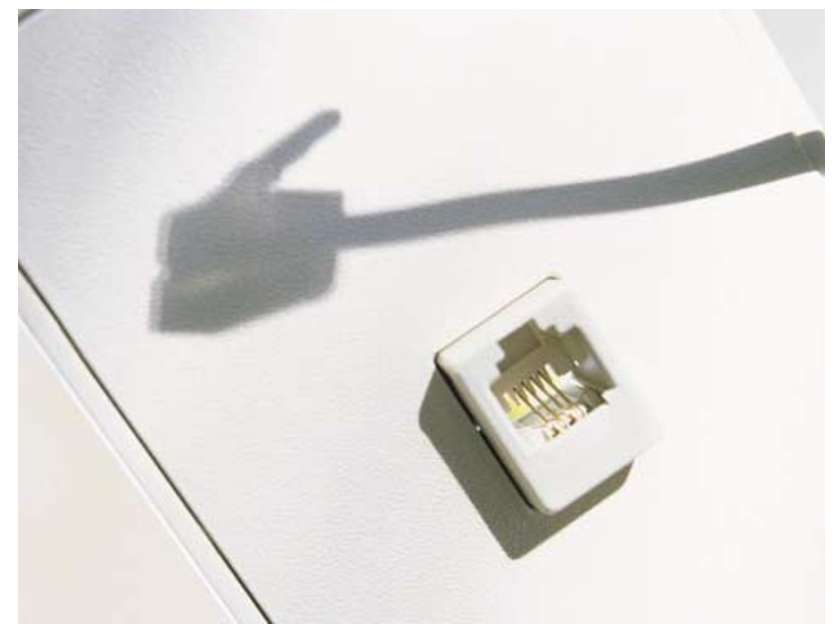

TUMOUR SUPPRESSORS

\section{ALL from a lost connection}

Although adaptors are useful gadgets for connecting unrelated objects, all sorts of problems arise when they get damaged or lost. Adaptor molecules have an important role in immune regulation - directly influencing $B$-cell maturation and the development of tolerance - but as Jumaa et al. report in Nature, loss of the B-cell-specific adaptor SLP-65 is associated with a type of acute lymphoblastic leukaemia (ALL).

ALL is the most common form of childhood cancer and often arises from a block in the B-cell development programme that occurs as cells differentiate from precursor (pre-) B cells to mature cells. Mice lacking Slp-65 have more pre-B cells and fewer mature $B$ cells than wild-type mice, and $5-10 \%$ develop pre-B-cell leukaemia. In vitro, Slp $65^{-1-}$ cells also have a greater proliferative capacity. These data indicate that SLP-65 might limit proliferation of pre-B cells and promote differentiation into mature cells. To test this, the authors introduced either Slp- 65 or a Slp- 65 mutant into a Slp $65^{-1-}$ pre-B cell line - only the wild-type Slp- 65 was able to induce differentiation into mature $B$ cells. Also, on injection into immune-deficient mice, the cells expressing mutant Slp-65, but not wild-type Slp-65, caused pre-B-cell leukaemia. So, as human pre-B ALL has a similar phenotype, might it also have a similar defect in SLP-65 expression?

The authors tested bone marrow from 34 pre-B ALL patients and found that $47 \%$ had a complete loss or pronounced reduction in SLP-65 expression. This confirms that human pre-B ALL resembles mouse $S l p 65^{-1-}$-induced pre-B-cell leukaemia, but what is causing this decrease in SLP-65? Analysis using reverse transcriptase polymerase chain reaction revealed that SLP-65-deficient B cells express aberrant SLP65 mRNA transcripts containing an additional sequence between exon 3 and 4 of SLP65. Sequence analysis of this region of SLP65 revealed two alternative exons containing premature stop codons that - if incorporated into SLP- 65 mRNA by alternative splicing - interrupt the SLP-65 open reading frame. These findings indicate that SLP-65 deficiency might be a primary cause of pre-B ALL and improve our understanding of the mechanisms that underlie this disease.

Emma Croager

(2) References and links

ORIGINAL RESEARCH PAPER Jumaa, $H$. et al. Deficiency of the adaptor SLP-65 in preB-cell acute lymphoblastic leukaemia. Nature 423, 452-456 (2003) FURTHER READING Flemming, A. et al. The adaptor protein SLP-65 acts as a tumour suppressor that limits pre-B cell expansion. Nature Immunol. 4, 38-43 (2003) WEB SITE

Michael Reth's lab:

http://www.immunbio.mpg.de/groups/reth/reth_html.html

\section{TRIAL WATCH}

\section{MRI for early detection}

Magnetic resonance imaging (MRI) is currently used to monitor the response to therapy in patients with stage-known breast cancer. Data presented at the American Society of Clinical Oncology annual meeting provide evidence that MRI is also useful for the early detection of breast cancer in women with high risk of the disease.

Results of the largest non-randomized, prospective, multicentre study to compare different breast cancer screening methods were presented by Jan Klijn (Erasmus Medical Center, Rotterdam). More than 1,000 women with a $15 \%$ or greater familial or genetic lifetime risk for breast cancer were enrolled. The women with genetic risk had a mutation in the BRCA1 or $B R C A 2$ gene. They were evaluated by clinical breast examinations, yearly mammography and yearly MRI.

Over two years, 40 breast cancers were found $-46 \%$ of these were less than $1 \mathrm{~cm}$ in size and most had not spread to the lymph nodes. Clinical breast examination identified $16 \%$ of the tumours and mammography identified $36 \%$. MRI proved to be a much more sensitive technique, identifying $71 \%$ of the tumours. MRI can pick up tumours that mammography would miss, such as ductal carcinoma in situ, even if the tissue is dense - as it often is in younger women - or if the tumour is not calcified. Although MRI was less specific than mammography $88 \%$ compared with $95 \%$ - the investigators recommend the routine use of MRI in addition to mammography in women with $B R C A 1$ or $B R C A 2$ mutations. Another consideration is that women with familial breast cancer are more susceptible to the mutagenic effects of the lowdose irradiation used for a mammogram, so exposure to X-rays should be kept to a minimum.

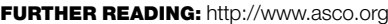

\section{Multitargeting}

The effectiveness of most targeted anti-angiogenic agents has been disappointing, but at the recent American Society of Clinical Oncology meeting, exciting data were presented from Phase I trials of a multitargeted receptor tyrosine kinase inhibitor, SU-11248. This small-molecule drug targets vascular endothelial growth factor (VEGF) receptor, platelet-derived growth-factor receptor (PDGFR), KIT and FLT3 receptors, all of which are involved in signalling pathways that are key to tumour angiogenesis.

One study included 41 patients with a variety of solid tumours. Although this was a dose-finding study, antitumour activity was seen — four patients had a partial response and 26 patients had stable disease. Decreased levels of VEGF receptor and increased plasma levels of VEGF were seen within 2 weeks of treatment in the responding patients. The investigators noticed some tumour regrowth between cycles of therapy, which they hypothesized might be due to increased VEGF ligand and receptor expression.

Another study was carried out in patients with gastrointestinal stromal tumours (GISTs) that were resistant to imatinib - the small-molecule drug that targets BCR-ABL, KIT and PDGFR. The trial of SU-11248 in GIST is ongoing, and the investigators are collecting information on the genotype of the tumours, and the expression and phosphorylation status of KIT and PDGFR. They are monitoring biomarker biological response by positron emission tomography (PET) and are also looking for markers of exposure, response and efficacy in the blood and plasma of patients. So far, responses, as measured by computed tomography (CT) scan, have been seen in 5 patients of the 32 enrolled, and these responses correlate with a decrease in KIT.

FURTHER READING: http://www.asco.org 